\title{
El desarrollo turístico de zonas rurales en España a partir de la creación de rutas del vino: un análisis DAF0
}

\author{
María Genoveva Millán Vázquez de la Torre* \\ María Genoveva Dancausa Millán \\ Universidad de Córdoba, España
}

\section{Resumen}

Ante la actual crisis económica, las zonas rurales de la Península Ibérica necesitan crear nuevas actividades que fomenten la generación de riqueza, proporcionando rentas que complementen sus ingresos procedentes del sector primario. La creación de rutas enológicas puede ser una solución para evitar la pérdida de empleos en estas comarcas tan debilitadas. El presente trabajo analiza las Rutas del Vino de España, en concreto la denominación de origen de Jerez (sur de España), mediante un análisis DAFO (debilidades, amenazas, fortalezas y oportunidades).

\section{Palabras Clave}

Gastronomía, desarrollo rural, rutas del vino, Península Ibérica. 


\title{
Creating rural tourism in Spain by developing wine routes: a SWOT analysis
}

\author{
María Genoveva Millán Vázquez de la Torre* \\ María Genoveva Dancausa Millán \\ Universidad de Córdoba, España
}

\begin{abstract}
Given the current economic crisis, rural areas of the Iberian Peninsula need new activities to be created that encourage wealth production, thus providing revenue to supplement income generated in the primary sector. Creating wine routes can be a solution to prevent the loss of jobs in these weakened regions. This work analyzes the Wine Routes of Spain, namely the denomination of origin of Jerez (southern Spain), using a SWOT analysis (strengths, weaknesses, opportunities, and threats).
\end{abstract}

\section{KEY WORDS}

Gastronomy, rural development, wine tours, Iberian Peninsula. 


\section{Introducción}

En las últimas décadas se ha observado un cambio en los gustos del consumidor turístico, por lo que, para responder a su demanda, se han creado nuevos segmentos de mercado con mayor oferta turística, dentro de la cual se encuentra el turismo gastronómico.

La gastronomía forma parte de la identificación cultural de un territorio; dado su carácter histórico y cultural, los alimentos se han convertido en recursos turísticos, y ya no sólo son vistos como facilitadores de la experiencia turística (Oliveira y Rocha, 2011). Si se formulan productos turísticos adecuados, el alimento no únicamente acrecienta el valor de un destino sino también el propio; entre territorio y alimento retoza la identidad local (Barrera y Bringas, 2008). La gastronomía, en cuanto recurso turístico, es apreciada tanto por su propio valor intrínseco como por su carácter simbólico, en la medida en que actúa como identificador de pueblos y territorios (Armesto y Gómez, 2004), puede ser analizada como un elemento del espacio natural y social, y ser percibida como un elemento relacionado con la producción y transformación del espacio regional (Mascarenhas y Gândara, 2010).

Se puede denominar turismo gastronómico a las visitas a productores primarios y secundarios de alimentos, festivales gastronómicos, restaurantes y lugares específicos donde la degustación de platos y/o la experimentación de los atributos de una región especializada en la producción de alimentos es la razón principal para realizar el viaje (Schlüter y Thiel, 2008). En el panorama turístico español, el turismo gastronómico es uno de los productos emergentes que más se está desenvolviendo en los últimos años; ha obtenido el apoyo de entidades públicas como la Secretaría General de Turismo en el marco "Calidad de los productos turísticos”, favoreciendo el desarrollo de las Rutas del Vino de España.

La Europa mediterránea posee una vasta tradición en materia de denominaciones de origen e indicaciones geográficas, distintivos que vinculan la calidad de un alimento a su origen geográfico. Las rutas gastronómicas constituyen un interesante instrumento para posicionar los productos y asociarlos a un apelativo de calidad geográfica (Barrera, 2006). Dentro de estas rutas hay que destacar las del vino: recorridos señalados y publicitados con paneles especiales en los cuales se insiste en los valores naturales, culturales, medioambientales, viñedos y bodegas, explotaciones agrícolas individuales o cooperativas abiertas al público; estas rutas son 
el medio a través del cual los territorios vitícolas y sus producciones relacionadas pueden ser divulgados, comercializados y disfrutados en forma de oferta turística.

El concepto de ruta del vino consiste en constituir en determinados destinos rurales con orientación clara hacia el turismo cultural y rural un producto integrado basado en las actividades vitícolas, gastronómicas y culturales, acompañado por una amplia oferta de alojamiento con servicios relacionados con esas actividades (enotecas, vinoteras, museos del vino, mueblerías especializadas), así como por una extensa oferta complementaria, por ejemplo golf, hípica, comercios, restaurantes, vida nocturna (López, 2010).

Una ruta del vino no es solamente la señalización: son horarios seguros para las visitas a las fincas, la capacidad de guiar y mostrar su propia empresa, saber acoger y dar la bienvenida a un turista, la modificación estructural de la bodega, cuidar la limpieza y la oferta de la venta. Según Bruwer (2003), una ruta del vino se caracteriza por la mezcla de agentes naturales, culturales y sociales, además de actividades en bodega, la gastronomía local, actividades de observación del mundo del vino (como pueden ser los museos) y compra de productos locales artesanales y diferenciados. Es una ruta o visita por todos los lugares y recursos que tienen que ver con el vino o su estilo de vida (Ravenscroft y van Westering, 2001), sin desvirtuar o hacer uso excesivo de este estilo de vida, dando lugar a ficciones o simulación.

El turismo del vino presenta una completa experiencia sensorial (Getz, 2000): el visitante puede experimentar el placer del sabor, del olor, del tacto, de la vista y del sonido. Cualquier enoturista, con independencia de sus conocimientos de enología, aprecia el paladar de los diferentes vinos, el olor de las bodegas, la manera de coger una copa para catar un vino, la visión de una bodega, a veces centenaria, o el sonido de abrir una botella de buen vino.

En este sentido, la clusterización de actividades y atracciones, y el desarrollo de rutas turísticas, estimula la cooperación y relación entre las distintas empresas de las áreas rurales. Por tanto, el turismo es una alternativa para llevar a cabo estrategias de desarrollo y regeneración económica y social de estas áreas, siendo las cooperativas una de las formas organizativas con más futuro en el campo del turismo rural (y, por ende, en el enológico), debido a que se pueden adaptar más rápidamente a las exigencias de la demanda (Fernández y Puig, 2002). El turismo del vino, como modelo de desarrollo económico sostenible e integrador de determi- 
nadas áreas, es capaz de dinamizar la competitividad de un territorio, incrementar y mejorar la producción vinícola, respetar el medio ambiente y mejorar las condiciones de vida de los ciudadanos.

España, uno de los grandes productores de vino del mundo, ha centrado su producción vinícola generalmente en pequeñas y medianas empresas, muchas de ellas con una estructura organizativa de cooperativa, lo que ha generado riqueza suficiente para frenar en parte los flujos migratorios hacia las grandes ciudades. Sin embargo, en los últimos años, las empresas vinícolas han cambiado radicalmente su forma organizativa, evolucionando hacia vinos de mayor calidad que han originado también mayores beneficios (Martínez-Carrasco, Brugarolas y del Campo, 2005). Asimismo, se encuentran con una incipiente diversificación de la generación de sus ingresos a través de, entre otras opciones, la creación de rutas turísticas enológicas, las cuales tienen ya una gran aceptación en otros países, pero en España apenas comienzan. Quizá de este exiguo desarrollo surja una serie de inconvenientes que se dibuja en la oferta turística de este producto, como el bajo nivel de desarrollo turístico en el entorno rural, la escasa oferta complementaria relacionada con el mundo del vino, la falta de una adecuada promoción de los recursos enoturísticos existentes que permitan su estructuración y puesta en valor, o que el turista apenas pernocta en el área geográfica donde se localizan las bodegas, con lo cual su gasto en la zona es muy bajo.

El objetivo de este trabajo es presentar una revisión de la literatura elaborada en el campo del enoturismo y mostrar cómo se vertebra la actividad complementaria en la ruta del vino correspondiente al Marco de Jerez (en el sur de España), con un análisis DAFO (debilidades, amenazas, fortalezas y oportunidades).

\section{Antecedentes}

La definición y conceptualización del término enoturismo no es uniforme, ya que se puede analizar desde distintas perspectivas, como la del marketing o la motivación de los viajeros. Por ello, y siguiendo a Getz y Brown (2006), el enoturismo es simultáneamente un comportamiento del consumidor, una estrategia para desarrollar el área geográfica y su mercado del vino, y una oportunidad de promoción de las bodegas para vender sus productos directamente a los consumidores. En este 
sentido, Mitchell y Hall (2006) identifican siete campos diferentes en el estudio del enoturismo: el propio producto turístico y su desarrollo, el desarrollo regional con base en el enoturismo, el tamaño de las bodegas objeto de la visita, la segmentación del enoturista, el comportamiento del turista, el análisis de la experiencia del visitante, y un último campo de investigación sobre los riesgos, para los visitantes, relacionados con los controles de calidad sanitarios.

El enoturismo se configura en los últimos años como una vía complementaria de generación de empleo y de riqueza en zonas rurales. La creación de nuevos destinos competitivos distintos de los tradicionales de sol y playa permite un incremento de las rentas en las zonas de interior y evita la migración de la población de las áreas rurales. De tal modo, Hall et al. (2000) examinan el desenvolvimiento y gestión del turismo del vino en el mundo y su incidencia en el desarrollo de las diversas zonas donde se asienta. Por su parte, O’Neill y Charters (2000) señalan que el enoturismo atrae a un turista ético y responsable que interactúa bien con los residentes y gasta importantes sumas de dinero, mientras que Carlsen (2004) profundiza en la interrelación entre turismo y vino.

En las últimas dos décadas, el enoturismo ha tenido un gran impulso en la literatura científica; así lo documentan investigaciones en Australia (Jolley, 2002), Canadá (Hashimoto y Telfer, 2003), Chile (Egan y Bell, 2002; Sharples, 2002), Francia (Frochot, 2000), Grecia (Tzmitra-Kalogianni, Papadaki y Alexaki, 1999), Hungría (Szivas, 1999), Italia (Brunori y Rossi, 2000), Nueva Zelanda (Beverland, 1998; Hall et al., 1998), Portugal (Correia, Passos y Charters, 2004; Marques, 2006), Sudáfrica (Bruwer, 2003) y España (Gilbert, 1992; Hall y Mitchell, 2000). Los primeros trabajos científicos en España abordaban principalmente la zona de La Rioja, los cuales se han completado con el estudio de otras zonas vinícolas como Priorato (Armesto y Gómez, 2004) o Montilla-Moriles (Millán y Melián, 2008).

En Europa, el enoturismo se ha desarrollado sobre todo a partir de rutas turísticas, caracterizadas por atractivos tanto naturales (montañas y otros paisajes) como creados (bodegas, museos del vino, etc.). Asimismo, una ruta es una mezcla de aspectos culturales, medioambientales y sociales (Bruwer, 2003). Los componentes de la experiencia del turista en una ruta del vino son, entre otros, la visita a la bodega, la contemplación de un viñedo, la entrada a un museo, la degustación de la gastronomía propia de la zona, la visita a un centro de información vitivinícola, la contemplación de paisajes, la compra de vino y el alojamiento en lugares vinculados 
con este tema (Ravenscroft y van Westering, 2001). En definitiva, la ruta turística enológica es la suma del esfuerzo de muchas empresas públicas y privadas.

Para crear una ruta turística del vino es imprescindible establecer una serie de alianzas entre las diversas empresas encargadas de prestar los servicios correspondientes, ya que, recordemos, en la actividad turística el viajero (consumidor) sólo compra un producto (satisfacer una necesidad a través de un viaje), mientras que este producto único es vendido por varios oferentes (por ejemplo, el hotelero, el restaurador y el bodeguero). Por ello resulta imprescindible instituir bases, a veces incluso de carácter jurídico, con el propósito de cerrar acuerdos o alianzas entre los oferentes -como la alianza en la zona de Ontario (Canadá) entre 27 bodegas para intentar incrementar 50 \% la demanda de visitantes a corto plazo (Telfer, 2001).

Una vez creada la ruta turística, el siguiente paso sería promocionarla con la finalidad de llegar a su potencial demandante, y aquí surge una pregunta clave: ¿quiénes son los turistas del vino? Existen varios estudios que analizan la segmentación de estos turistas, entre los cuales destaca el realizado por Charters y Ali-Knight (2002), quienes los agrupan en cuatro tipologías diferentes (figura 1):

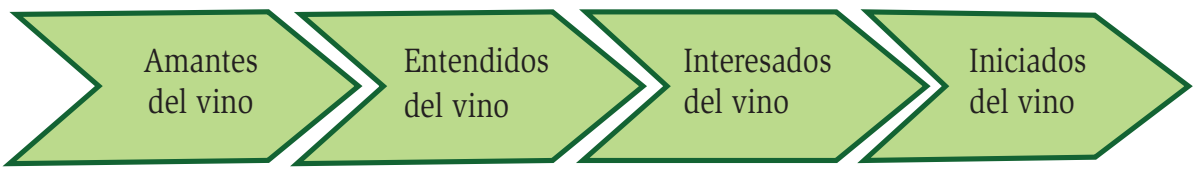

Fuente: Elaboración propia.

Figura 1. Perfil del enoturista según Charters y Ali-Knight

- Amantes del vino. Son aquellos que tienen una vasta educación en temas enológicos; la principal motivación de su viaje es catar diferentes tipos de vino, comprar botellas y aprender in situ; además, están muy interesados en la gastronomía local.

- Entendidos en el vino. Son aquellos que aunque no tienen una vasta educación en temas enológicos, conocen relativamente bien el mundo del vino; suelen tener una formación universitaria y la motivación principal de su viaje es llevar a la práctica lo que han leído en revistas especializadas. 
- Interesados en el vino. Son aquellos que no tienen una formación técnica en temas enológicos pero les interesa este mundo; la motivación principal de su viaje es conocer algunas bodegas, aunque no con carácter exclusivo sino como complemento de otras actividades.

- Iniciados en el vino. Son aquellos que por diferentes razones (como la publicidad de una ruta o querer probar nuevas experiencias) se acercan a una bodega sin tener ningún tipo de conocimiento en este campo; la motivación principal de su viaje es otra totalmente distinta, pero aceptan, de forma secundaria, pasar unas horas visitando una bodega, donde realizan compras destinadas al consumo particular o, en la mayoría de las ocasiones, para regalar.

En conclusión, si se identifican los diversos tipos de cliente, se le puede brindar a cada uno una oferta muy específica, más acorde con sus gustos.

\section{Las rutas del vino en España}

El sector vitivinícola español es de gran importancia tanto por el valor económico que genera como por la población que ocupa y por el papel que desempeña en la conservación medioambiental. España, con 1.16 millones de hectáreas (ha) destinadas al cultivo de la uva $(97.4 \%$ destinado a vinificación, $2 \%$ a uva de mesa, $0.3 \%$ a la elaboración de pasas y el $0.3 \%$ restante a viveros), sigue siendo el país con mayor extensión de viñedo de la Unión Europea y del mundo (representa 25 \% de la superficie total de la Unión Europea -seguido por Francia e Italia con aproximadamente 18 \% cada uno- y del mundo, con 14.5\%). Su tradición elaboradora de vinos se remonta a la época de los romanos. La vid ocupa el tercer lugar en extensión de los cultivos españoles, detrás de los cereales y el olivar.

La situación geográfica, las diferencias climáticas y la variedad de suelos hacen de la Península Ibérica un lugar privilegiado para la producción de vinos de características muy distintas. Se cultiva viñedo en las 17 comunidades autónomas en las que se divide España, si bien cerca de la mitad de la extensión total se encuentra en Castilla-La Mancha (528 000 ha y 50.4 \% del viñedo plantado), la zona geográfica con mayor extensión dedicada a su cultivo en el mundo, seguida de Extremadura (cerca de 88000 ha, 8.1\%), Valencia (76 000 ha), Castilla y León (72 000 ha), 
Cataluña, Aragón, Murcia, Andalucía y La Rioja, siendo esta última la comunidad autónoma que dedica, proporcionalmente a su superficie cultivada, mayor extensión al cultivo del viñedo. La media de explotación agraria en España es de 3.34 ha, aunque varía entre las distintas regiones; las explotaciones más pequeñas se dan en Galicia, las mayores, en Murcia. Con ayuda de la Organización Común del Mercado Vitivinícola (OCM) del vino, en la campaña 2009-2010, se han arrancado 50 ha de viñedo.

La producción de vino en el país, según los datos del Fondo Español de Garantía Agraria (FEGA), ascendió en 2010 a 34.7 millones de hectolitros (hl): casi $19.5 \mathrm{mi}-$ llones de vinos de mesa, 2.5 millones de vinos con indicación geográfica protegida (IGP) y 13.2 millones de vinos de calidad de denominación de origen (DO).

En cuanto al reparto geográfico de la producción, se tiene lo siguiente:

- Castilla-La Mancha continúa siendo la principal región productora con casi $50 \%$ del total, a pesar del fuerte descenso que experimentó en su producción (17.2 millones de hl). De este volumen, 12.1 millones corresponden a vinos sin IGP, 2.9 a vinos con IGP y 2.1 a vinos con denominación de origen protegida (DOP).

- Cataluña se sitúa en segundo lugar en cuanto a producción, con 3.1 millones de hl, que representan $9.2 \%$ del total nacional, y 2.8 millones de hl corresponden a vinos con DOP. En esta comunidad autónoma, la producción de vino blanco duplica a la de tinto y rosado.

- Extremadura, con 2.8 millones de hl, es la tercera comunidad productora de vinos $(9.1 \%)$. En esta zona, la producción de vino blanco también supera la de los tintos.

- La Comunidad Valenciana ocupa el cuarto lugar, con una producción de 2.3 millones de hl, $6.8 \%$ del total, y $12.5 \%$ más que en 2008 .

- La Rioja se ubica en quinto lugar, con 5.9 \% del total nacional, lo que supone 2.07 millones de hl y $1.5 \%$ más que en la campaña precedente.

- El sexto lugar entre las comunidades autónomas productoras es para Castilla y León, con 1.6 millones de hl, lo que supone 4.7 \% de la producción nacional con un incremento de $17.5 \%$ respecto al año anterior.

- Aragón se encuentra en el séptimo sitio, con casi 1.30 millones de hl, 3.7 \% del total y $25 \%$ de incremento sobre la campaña anterior. 
- A Andalucía le corresponde el octavo lugar, con 1.16 millones de hl, lo que representa $3.3 \%$ del total nacional y - $10 \%$ respecto a la campaña precedente.

- Por último estarían, con volúmenes más pequeños, las comunidades de Navarra (2.5\%), Murcia (2.2\%), País Vasco (1.8 \%) y Galicia (1\%).

Según se expone en la Ley 24/2003, de 10 de julio, de la Viña y del Vino, los vinos elaborados en España podrán acogerse a alguno de los siguientes niveles, dependiendo de los requisitos que cumplan y, en su caso, de conformidad con la legislación autonómica:

a) Vinos de calidad producidos en una región determinada (VCPRD), en los que, a su vez, podrán establecerse los siguientes niveles:

- Vinos de calidad con indicación geográfica

- Vinos con denominación de origen

- Vinos con denominación de origen calificada

- Vinos de pagos

b) Vinos de mesa:

- Vinos de mesa con derecho a la mención tradicional "vino de la tierra"

- Vinos de mesa

La Secretaría de Estado de Turismo (2000) define la ruta del vino como "la integración bajo un mismo concepto temático de los recursos y los servicios turísticos de interés, existentes y potenciales, de una zona vitivinícola, planteados desde la autenticidad y la vocación vivencial, con el fin de construir un producto desde la identidad propia del destino, que facilita la comercialización conjunta de toda la zona y garantiza el nivel de satisfacción de la demanda, impulsando así el desarrollo económico-social integral de la misma”.

La Asociación Española de Ciudades del Vino (Acevin) y la Secretaría General de Turismo recogen en el Manual del Producto Turístico Rutas del Vino de España la metodología para la implantación de este producto turístico y su posterior certificación por parte del Comité de Gestión y Certificación de las Rutas del Vino de España.

La estrategia de la Acevin es valorizar el potencial endógeno de las zonas y ciudades donde el cultivo y la elaboración del vino han sido y son la base productiva en torno a la cual se han configurado históricamente. Las ciudades del vino promueven 
la valorización de este sector con objeto de potenciarlo como motor de otras actividades (desarrollo turístico, promoción económica, empleo, etc.). La presencia del vino en cuanto base productiva agroindustrial actúa como eje articulador de actividades diversas: a las labores tradicionales vitícolas y vinícolas de cultivo, elaboración y distribución del vino se agrega un conjunto de actividades conexas y complementarias:

a) Estímulo y desarrollo de las actividades turísticas:

Marketing turístico

Museos del vino

Restauración y actividades culinarias

Hoteles y alojamientos

Tiendas especializadas y pequeño comercio

Rutas del vino

b) Promoción cultural del territorio:

Recuperación y promoción del patrimonio arquitectónico e histórico

Fiestas populares

Rehabilitación del patrimonio arquitectónico

c) Actividades comerciales:

Publicaciones, organización de ferias, actos

Técnicas informáticas de comercialización y promoción

d) Protección de los recursos naturales:

Depuración y reciclaje de agua

Protección de zonas naturales

Producción biológica

Ordenación del territorio

De 2001 a la fecha, la Secretaría General de Turismo, a través del Programa Calidad en los Productos Turísticos, ha prestado asistencia técnica a destinos piloto para diagnosticar la situación y evaluar los recursos, crear el producto turístico Rutas del Vino de España e implantarlo. El proyecto se inició en 2001 con seis destinos piloto -El Penedés, Jumilla, La Mancha, Montilla-Moriles, Rías Baixas y 
Utiel-Requena-, y en diez años se ha podido consolidar una marca turística de calidad que es referente nacional: el enoturismo o turismo del vino, pasando de seis destinos enoturísticos a un Club de Producto Turístico integrado por 22 rutas del vino, 16 de las cuales están certificadas conforme a la marca Rutas del Vino de España: Ruta del Vino de Bullas (Murcia), Jumilla (Murcia), Camino del Vino de La Mancha, Marco de Jerez, Ruta del Vino de Montilla-Moriles, Navarra, Rías Baixas, Rioja Alavesa, Somontano (Aragón), Tacoronte-Acentejo (Canarias), O Ribeiro (Galicia), Ribera de Duero, Utiel-Requena (Comunidad Valenciana), Campo de Borja (Aragón), Lleida (Cataluña) y Ruta del Penedés (Cataluña), y seis se encuentran en proceso de certificación: Ruta del Vino de La Rioja Alta (Rioja), Condado de Huelva (Andalucía), Ycoden-Daute-Isora (Canarias), Alicante, El Bierzo (Castilla y León) y Ribera del Guadiana (Extremadura). Asimismo, las Rutas del Vino de España están incluidas dentro de la oferta turística que se promociona en el extranjero a través de los canales de Turespaña, como la < www.spain.info >, las oficinas españolas de turismo, etcétera.

Rutas del Vino de España trae asociados beneficios potenciales de diversa naturaleza que constituyen el valor añadido que este producto tiene con respecto a otras ofertas de turismo enológico y gastronómico (Acevin):

- Para el cliente o visitante. El cliente obtiene sinergias en cuanto a poder conocer el entorno de elaboración del producto, y recibe en definitiva un servicio más amplio y profesional, respaldado por una institución con más fuerza y prestigio que cada una de las empresas individuales que la constituyen.

- Para los empresarios. Supone la creación de una marca conjunta fuerte y distintiva en el mercado, asistencia mutua y colaboración, explotación de un nicho de mercado creciente y dinámico, desestacionalización de la demanda, introducción de sistemas de calidad de servicio, formación, central de compras y de ventas, promoción y comercialización conjunta.

- Autorregulación del sistema. Los ayuntamientos, consejos reguladores y empresarios implicados en la ruta del vino adquieren el principal protagonismo en la conformación y puesta en marcha del producto. 
El turismo del vino impulsa el desarrollo socioeconómico integral y sostenible del territorio generando empleo, riqueza, y mejorando la calidad de vida de la población local; fomenta la actividad turística en las Do del vino, posibilita el surgimiento de nuevas vías de ingresos complementarios para los productores vitivinícolas y aumenta las ventas de vino.

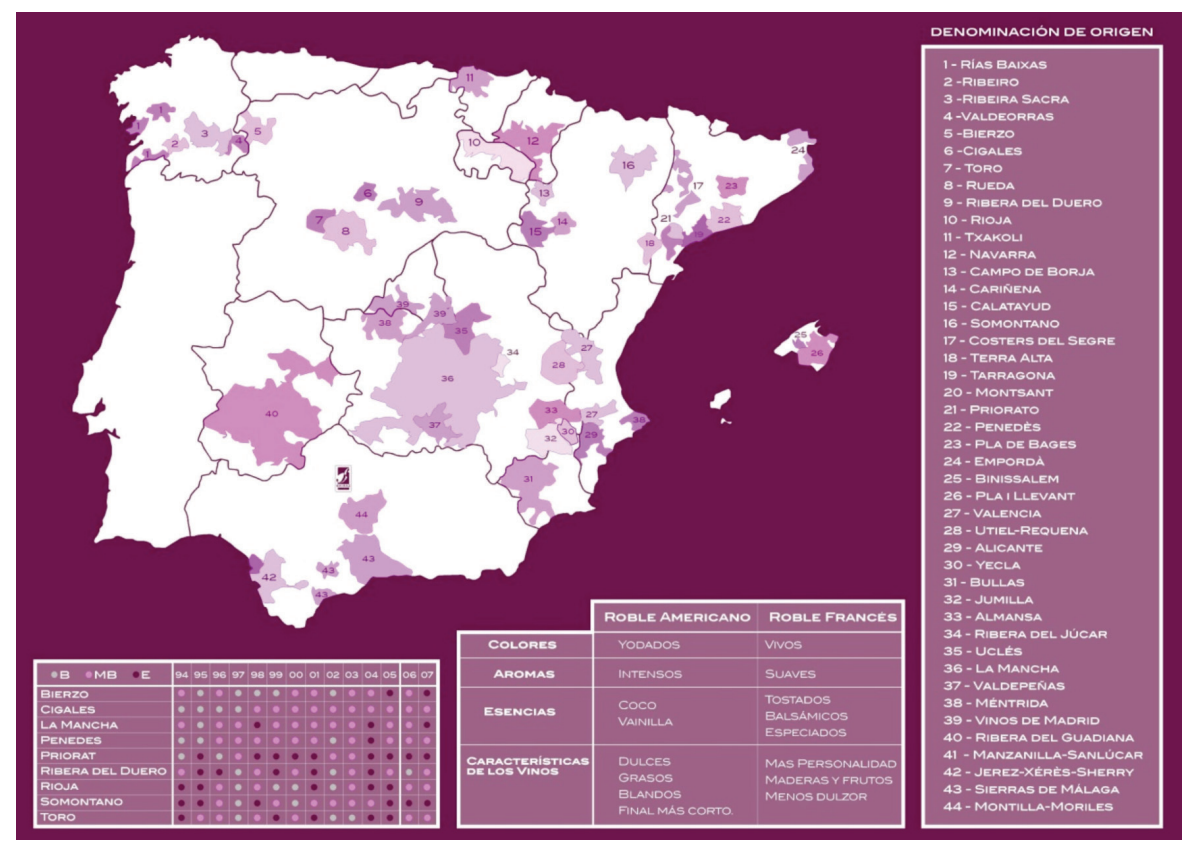

Fuente: Observatorio Turístico de las Rutas del Vino de España.

Figura 2. Denominaciones de origen del vino en España

La metodología para la implantación de este tipo de producto turístico se realiza en tres fases: de lanzamiento, de desarrollo y de consolidación (Secretaría de Estado de Turismo, 2000). La primera comienza cuando las entidades públicas y privadas implicadas en el producto turístico (establecimientos hoteleros, administraciones públicas, bodegas, etc.) crean un ente gestor de la ruta y adecuan las instalaciones y servicios de los establecimientos adheridos a la ruta con los requisitos de calidad exigidos por la Secretaría General de Turismo. Esta fase termina con el otorgamiento de un certificado como Ruta del Vino. 
La fase de desarrollo dura un año y se inicia una vez conseguida la certificación como Ruta del Vino. En ese lapso, el ente gestor pone en marcha las actuaciones detalladas por la Secretaría General de Turismo. Si el proceso de certificación finaliza con una valoración satisfactoria, el destino mantendrá su certificación como Ruta del Vino de España y pasará a la siguiente etapa; si, por el contrario, el proceso da una valoración negativa, el destino perderá su certificación como Ruta del Vino y será penalizado impidiendo que pueda volver a solicitar la certificación durante un año.

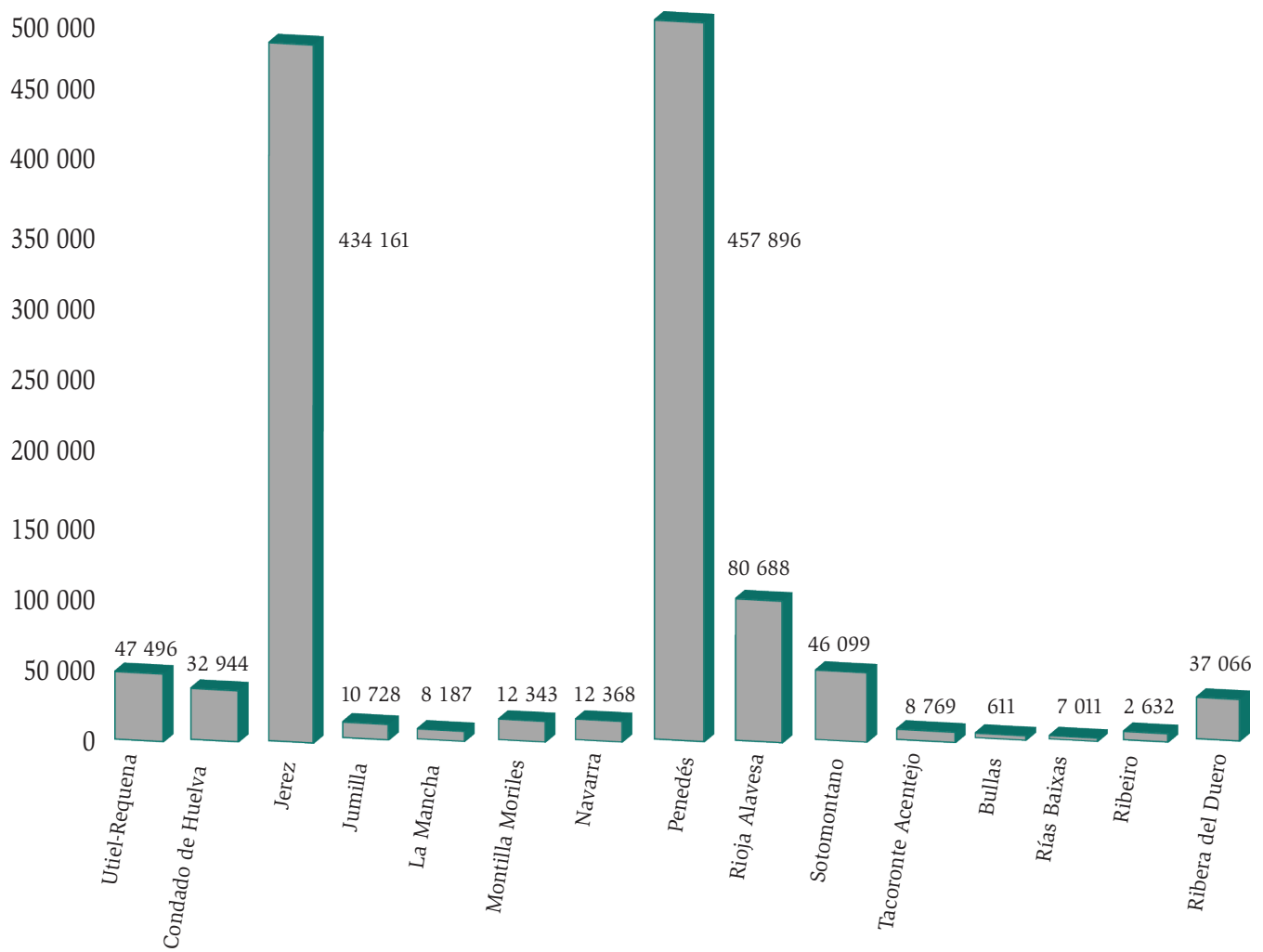

Fuente: Observatorio Turístico de las Rutas del Vino de España. 
La fase de consolidación consiste en un continuo proceso de comprobación de que la ruta mantiene el grado de exigencia tanto en lo referente a los establecimientos adheridos como a las actuaciones del ente gestor. Si en uno de estos procesos de comprobación se realiza una valoración negativa, se daría un plazo de seis meses para resolverlo y conseguir, de nuevo, un nivel mínimo. Tras ese tiempo, si el destino ha conseguido tal nivel mantendría su marca de Ruta del Vino, pero, de no ser así, la perdería y durante el año siguiente no podría solicitar la certificación.

Actualmente, según los estudios del Observatorio Turístico de las Rutas del Vino de España, la que más visitantes ha tenido (457 896) ha sido la Ruta del Vino y del Cava del Penedés (Enoturisme Penedès), seguida de la Ruta del Vino y Brandy del Marco de Jerez (431 161 visitantes). Cabe comentar que ambas zonas enoturísticas cuentan con algunas de las bodegas más concurridas del mundo, como Codorniu y Freixenet en Penedés o González Byass Tío Pepe en el Marco de Jerez (entre ambas superan los 100000 visitantes anuales). Rutas como éstas se encuentran en la fase de consolidación, mientras que aquellas con el menor número de turistas están en la fase de desarrollo (véase gráfica 1).

\section{Enoturismo y desarrollo local en España. Análisis de la oferta}

En los apartados anteriores se han indicado las características necesarias para la creación y el mantenimiento de las rutas del vino en España, tomándose como ejemplo una ruta consolidada con gran afluencia de turismo: la del Marco de Jerez.

Con base en el análisis de la repercusión del enoturismo en España, y según el informe del Observatorio Turístico de las Rutas del Vino de España en 2010, en 13 rutas certificadas $52 \%$ de las empresas que forman parte de la ruta del vino (bodegas, alojamientos, restaurantes, enotecas y comercios, museos y centros de interpretación, oficinas de información turística, etc.) considera que su adhesión a ella ha sido favorable, teniendo una actitud positiva ante la misma. De las empresas encuestadas, $51 \%$ tuvo más clientes tras incorporarse a la ruta, frente a $43.5 \%$ que indica que recibe el mismo número.

Respecto a la generación de empleo, 44 \% de las empresas creó un puesto de trabajo o más a partir de su adhesión a la ruta. Este dato confirma que el enoturismo puede generar riqueza, ya que crea empleo directo en épocas de crisis (gráfica 2). 
Pero también se promueve el empleo indirecto, ya que $56.6 \%$ de las empresas realizó inversiones para adaptar sus establecimientos al enoturismo, especialmente

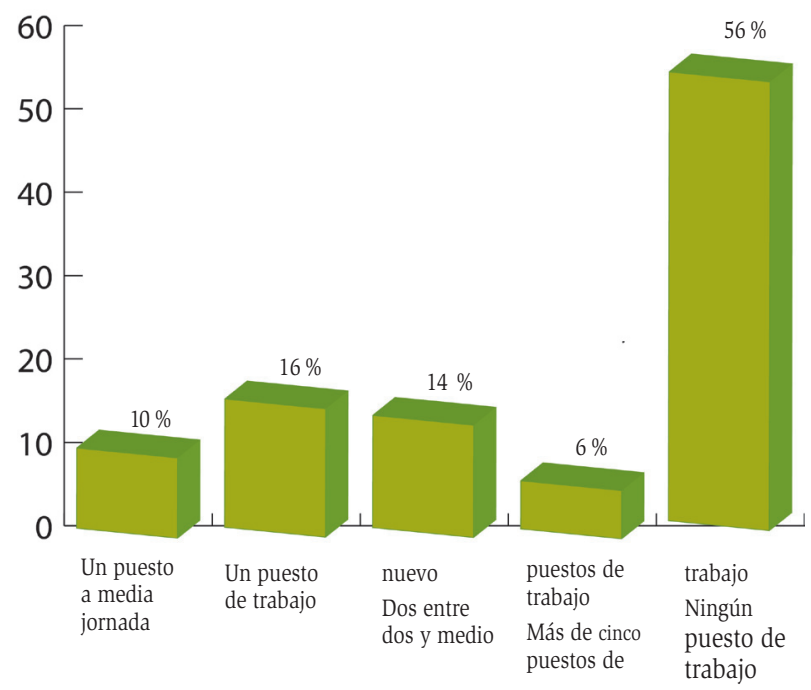

Fuente: Elaboración propia a partir de datos del Observatorio Turístico de las Rutas del Vino de España.

Gráfica 2. Creación de empleo en las empresas de las rutas del vino tras adherirse a ellas

obras de construcción y mejora, con lo cual se crearon puestos de trabajo en el sector de la construcción.

Hay que destacar que $30.6 \%$ de los alojamientos integrados en las rutas del vino son de tipo rural y por tanto el precio no es muy elevado; en $46 \%$ de los alojamientos el precio es inferior a 60 euros, con una estancia media de 2.35 noches, todavía por debajo de la estancia media en alojamientos rurales en España, que para 2009 fue de 3.01 noches.

Respecto a si es rentable para las empresas incorporarse a una ruta, $48.5 \%$ declara que sí, frente a $24.3 \%$ que dice que no (entre las ventajas que obtienen al adherirse a una ruta está el ahorro en costos de promoción, asistencia a ferias, etcétera). 
Se observa que las bodegas han ampliado su oferta y no se dedican exclusivamente a las visitas guiadas (gráfica 3 ). Todas las bodegas adscritas a una ruta realizan visitas guiadas con degustación; en 99 \% de ellas se puede comprar vino, lo que junto con el precio de la entrada que se cobra para ver la bodega genera ingresos adicionales a la fabricación del vino (el precio medio de la visita es de 3.92 euros). Así como hay rutas donde no se cobra nada, por ejemplo en la de UtielRequena, hay otras que tienen un precio elevado, como la del Marco de Jerez, con una media de 9 euros.

Otro factor importante es el nivel de satisfacción de las bodegas con su adhesión a una ruta: $56.1 \%$ está satisfecho o muy satisfecho, pues, como ya se ha indicado, el turismo del vino es un generador directo de ingresos por la venta del vino y otros servicios, y produce empleo y desarrollo en las zonas donde se ubican las rutas, porque se mejora la infraestructura y los servicios, como las carreteras o las vías de comunicación, beneficiando a los lugareños; además, mediante la publicidad de las rutas, también se están ofertando la cultura y la gastronomía de las zonas implicadas, así como actividades complementarias que reavivan las economías locales.

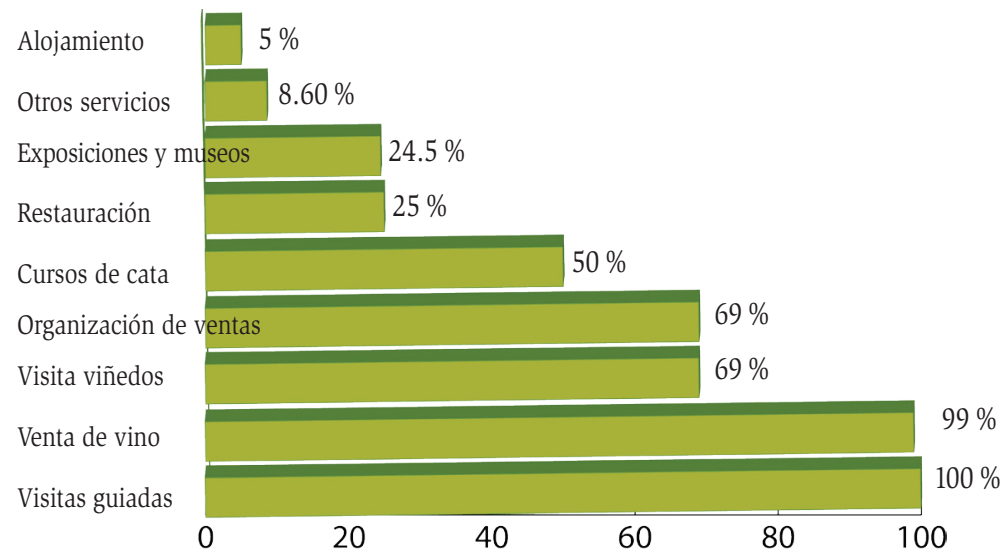

Fuente: Elaboración propia a partir de datos del Observatorio Turístico de las Rutas del Vino de España. 


\section{El enoturismo en la ruta del Marco de Jerez. Un análisis DAFo}

La ruta certificada del Marco de Jerez se originó en 2006, cuando se creó la Asociación Rutas del Vino y Brandy del Marco de Jerez. Cuenta con una superficie cultivada dedicada al viñedo de 10300 ha y engloba a las Do Jerez-Xérès-Sherry y Manzanilla-Sanlúcar de Barrameda. Está situada geográficamente en los términos municipales de Jerez de la Frontera, El Puerto de Santa María, Sanlúcar de Barrameda, Chipiona, Trebujena, Rota, Puerto Real, Chiclana de la Frontera y Lebrija, ofreciendo una rica variedad de paisajes y recursos enoturísticos que hacen posible nuevas experiencias.

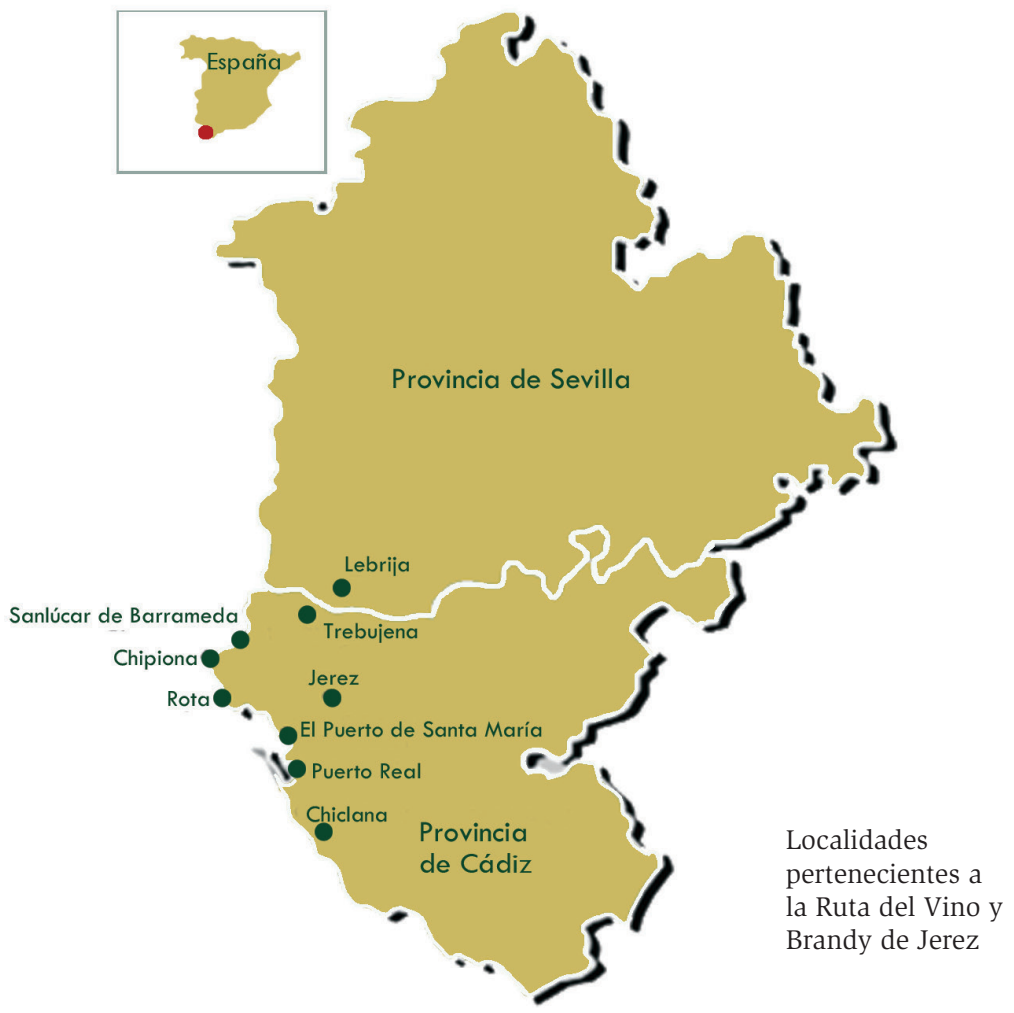

Fuente: Consejo Regulador del Marco de Jerez.

Figura 3. Poblaciones de la Ruta del Vino y Brandy de Jerez 
DEBILIDADES / WEAKNESSES

- Oferta de establecimiento desigual

- Inexistencia de centros de interpretación

- No existen estándares de calidad para los establecimientos turísticos

- Insuficiente conocimiento del turista del vino y su comportamiento

\section{FORTALEZAS /STRENGTHS:}

- Identificación del vino con la zona geográfica

- Amplia oferta de bodegas abiertas al público

- Rica oferta de actividades de ocio relacionadas con el vino

- Numerosa y variada oferta hotelera

- Buena red de comunicaciones

- Variada oferta complementaria

- Disponibilidad para desarrollar actividades todo el año

- Aumento asociacionismo en los municipios que forman parte de la ruta

\section{OPORTUNIDADES / OPPORTUNITIES}

\section{AMENAZAS / THREATS}

- Fuerte competencia en enoturismo por parte de otras regiones

- Intrusismo de personas ajenas al entorno del vino

- Ausencia de planificación turística adecuada

- Conversión del turismo en un "nuevo monocultivo" ca Marco de Jerez

- Creciente desarrollo y diversificación del mercado enoturístico mundial y nacional

- Aumento nivel de renta disponibles

- Tipo de población.

- Políticas públicas
- Buena imagen internacional de la comar-

Fuente: Elaboración propia.

Cuadro 1. Matriz DAFO (SWOT, por sus siglas en inglés) del turismo rural 
La Ruta del Vino y Brandy del Marco de Jerez, formada por más de 100 empresas asociadas entre bodegas, establecimientos de restauración, hoteles, museos, vinacotecas, etc., es la ruta de España con mayor número de socios. Sus vinos son muy conocidos y apreciados internacionalmente, sobre todo el vino blanco y dulce. Para certificar y velar por la calidad de sus caldos tiene un Consejo Regulador, cuyas funciones pueden resumirse en tres áreas fundamentales:

- control del origen y la calidad de los productos amparados,

- defensa del patrimonio común que suponen las denominaciones, y

- promoción y difusión de los productos protegidos por las mismas.

Dada la importancia de esta ruta, consolidada como la segunda en España por el número de turistas (más de 400 000) y como la primera por la cantidad de asociados, y al ser lo suficientemente representativa, fue escogida para realizar un análisis estratégico de las debilidades, amenazas, fortalezas y oportunidades (DAFO) del turismo del vino, que lo condicionan como generador de riqueza en Andalucía (véase cuadro 1).

Debilidades (Weaknesses): limitaciones que impiden que la zona vitivinícola haga frente a una amenaza o aproveche una oportunidad:

Desigual oferta de establecimientos en la ruta. La mayor concentración de los nueve municipios está en Jerez (50\% de las bodegas se encuentra en esa comarca, junto con $36 \%$ de oferta de alojamientos), pero también hay núcleos que aunque tengan bodegas no cuentan con alojamiento ni restaurantes asociados a la ruta. Entonces, si éstos no pueden atender las necesidades del enoturista, serán satisfechas en otras partes de la ruta, con la consiguiente pérdida de ingresos para aquéllos.

Insuficientes conocimiento del turista del vino y su comportamiento. Desconocimiento de los gustos y las motivaciones del turista, de qué lo impulsa a realizar esta ruta. Se requerirían estudios que analizasen qué demanda el enoturista.

Inexistencia de centros de interpretación. La ruta del Marco de Jerez recibe un número elevado de turistas extranjeros del segmento senior, por lo que sería necesario un centro de interpretación para atenderlos correctamente. 
Falta de estándares de calidad específica para los distintos establecimientos turísticos. En la ruta se observan tanto establecimientos con gran calidad y profesionalidad para atender al turista del vino -especialmente en determinadas bodegas (donde la visita turística se convierte en un placer de sentidos y gustos, captados por la atención del enoturista: "se mima al turista y se cuida hasta el último detalle”) y en algunos hoteles- como otros establecimientos, por lo general con pocos trabajadores, donde la atención al cliente es prácticamente nula.

Amenazas (Threats): factores del macroentorno que pueden afectar la capacidad de la zona para servir a sus mercados:

Fuerte competencia en enoturismo por parte de otras regiones. En especial La Rioja y Cataluña en España y el sur de Francia, Chile e Italia en el plano internacional, entre otros.

Intrusismo de personas ajenas al entorno del vino. En algunos casos las iniciativas turísticas en el ámbito rural están siendo desarrolladas por gente de fuera, sobre todo urbanitas sin ningún vínculo previo con el mundo rural, quienes ven la oportunidad de invertir en un sector que consideran en crecimiento y con grandes posibilidades. Esto puede producir un turismo rural desplanificado e incontrolable que conlleve costos irreparables, incluso más altos que el turismo tradicional de sol y playa, debido al mayor poder de penetración físico y psicosocial que posee.

Problemas derivados de la ausencia de planificación y control de las actividades turísticas. Por ejemplo, el abandono de actividades tradicionales, degradación de los espacios naturales, contaminación (acústica y de aguas), pérdida de la cultura autóctona de la zona, incremento de la delincuencia y el uso de drogas por influencia de los turistas, aumento del costo de la vida; en definitiva, problemas derivados de la falta de ordenación del territorio (Melián, Millán y López-Guzmán, 2008).

La actividad agraria tiende a ser una actividad de monocultivo, y existe poca diversidad agraria en cada zona. Por ello sería un error convertir el turismo rural en un "renovado monocultivo". No se trata de transformar a todos los empresarios agrarios del medio rural en operadores del sector turístico, pues 
el turismo rural no se entiende como la única solución para los problemas económicos de este medio, sino como un aporte económico y una forma de diversificación de rentas.

Fortalezas (Strengths): aspectos internos de las zonas rurales que les permiten aprovechar una oportunidad o hacer frente a una amenaza:

Identificación del vino con la zona geográfica. Al hablar de vino de Jerez se entiende como un caldo de alta calidad perteneciente a una comarca determinada.

Amplia oferta de bodegas abiertas al público. Éstas cuentan con personal cualificado para atender al enoturista.

Rica oferta de actividades de ocio relacionadas con el vino. Como vinoterapia, museos del vino, fiestas de la vendimia, y ferias anuales: la de Sanlúcar de Barrameda, dedicada al vino Manzanilla; la de El Puerto de Santa María, al vino fino, o la de Jerez de la Frontera, al caballo. Chipiona tiene unas fiestas en agosto en honor al Moscatel, mientras que Trebujena celebra al mosto, vino joven del año.

Numerosa y variada oferta hotelera. Desde departamentos y hoteles de dos estrellas hasta hoteles de cinco estrellas; el precio medio de la estancia se adapta a una gran diversidad de turistas.

Buena red de comunicaciones. Para acceder a la Ruta del Vino y Brandy del Marco de Jerez se cuenta con aeropuerto, autopista y una buena red ferroviaria, por lo que el enoturista puede desplazarse por cualquier medio de locomoción.

Otras actividades complementarias. Por ejemplo deportivas (tiene la mayor concentración de campos de golf de España, o también pueden llevarse a cabo actividades acuáticas), culturales (rico patrimonio histórico, yacimientos arqueológicos de fenicios), etcétera.

Disponibilidad para desarrollar actividades durante todo el año. Con ello se reduce la estacionalidad de la actividad turística en su conjunto, ya que la estancia media es la única variable que ofrece indicadores más altos en el turismo en espacios rurales que en el turismo en general. El número de días de 
estancia media de los turistas (3.6 días) es superior a la media de las rutas del vino (alrededor de 2.35). Sin embargo, esta modalidad turística se caracteriza por flujos de visitantes que realizan estancias breves -sobre todo, en los fines de semana- durante la mayor parte del año, aunque los máximos se producen en los meses centrales del verano.

Aumento del asociacionismo en los municipios que forman parte de la ruta. El apoyo entre empresarios y autoridades es imprescindible para desarrollar el sector turístico, pero aún necesita incrementarse; no obstante, sí existe respecto a las campañas publicitarias y de promoción de la ruta en folletos y ferias, con el consiguiente ahorro económico para quienes la integran.

Oportunidades (Opportunities): factores del macroentorno que si son aprovechados adecuadamente por la zona pueden proporcionarle ciertas ventajas $y / 0$ beneficios:

Buena imagen internacional de la comarca Marco de Jerez. En torno al clima, el paisaje, las playas, etcétera.

Creciente desarrollo y diversificación del mercado enoturístico mundial y nacional. Muchos enoturistas de la ruta de Jerez son extranjeros que vienen a realizar turismo de sol y playa y aprovechan unos días de su tiempo de ocio para hacer enoturismo, por lo que constituyen un mercado potencial bastante elevado.

Sinergias con otras modalidades de turismo. El enoturismo puede complementar otras opciones de turismo, como el deportivo, especialmente de golf (se observa la gran proliferación de campos de golf en esta zona y urbanizaciones de turismo residencial cercanas a ellos, utilizadas sobre todos por turistas extranjeros).

Tipo de población. Esta variable afecta directamente la demanda del sector turístico. Hoy en día se produce un creciente envejecimiento de la población y un aumento de la esperanza de vida, situación que se prevé que continuará durante algunas décadas. La pirámide de población de 2010 junto con la proyección de la pirámide poblacional para 2025 muestran un ensanchamiento en la parte superior y una tendencia a la inversión (mayor tamaño de la parte correspondiente a los 
grupos de edad superiores a 65 años). Esto puede beneficiar al sector turístico, ya que los colectivos con mayor estabilidad económica, adultos y de la tercera edad, son y serán mucho más que en décadas anteriores.

Aumento de los niveles de renta disponible. Esto ha ocurrido en los últimos años, lo que significa que una mayor parte de la renta familiar queda potencialmente disponible para vacaciones y viajes. En general, ante un mayor nivel de renta disponible se incrementa la demanda del turismo.

Adelanto de la jubilación de muchos trabajadores a una temprana edad. Este hecho, junto con otros logros laborables, ha suscitado la posibilidad de un mayor disfrute de tiempo libre. Asimismo, se ha mejorado la infraestructura, con la implantación y el desarrollo de nuevas tecnologías de la comunicación e información que favorecen el conocimiento de las zonas rurales y ayudan al progreso de esta alternativa de turismo.

\section{Conclusiones}

1. España cuenta con una amplia red de rutas del vino, algunas de ellas muy consolidadas y con un gran número de visitantes al año.

2. El turismo enológico es un generador de rentas complementarias en las zonas rurales, ya que crea empleo y por tanto riqueza.

3. Pertenecer a una ruta enológica conlleva una serie de obligaciones que deben cumplir las empresas que la conforman, para lo cual están los Consejos de las Denominaciones de Origen, que controlan la calidad del producto que ofrecen; esto se traduce en una mayor satisfacción y fidelización del cliente por el productor recibido.

4. La mayoría de las empresas que forman parte de una ruta del vino están satisfechas por ello, ya que obtienen ingresos más altos, sea por la venta de sus productos o por las actividades que realizan enfocadas al turismo.

5. No obstante el número de pernoctaciones del enoturista en la zona es bajo, en parte motivado por los horarios de apertura de las bodegas, los cuales varían mucho entre rutas, hay rutas donde casi todas las bodegas están 
abiertas al público por lo menos cuatro horas al día, mientras que otras sólo abren con previa cita o entre semana y no los fines de semana, lo que hace perder a potenciales clientes que podrían pasar más tiempo en la región.

6. La Ruta del Vino y Brandy del Marco de Jerez cuenta con grandes posibilidades para desarrollar los nueve municipios que engloba; entre sus aspectos positivos destaca una oferta variada en actividades relacionadas con el vino y actividades complementarias de ocio, lo que posibilita el aumento de las pernoctaciones del enoturista, además de que al estar identificado el producto vino con la zona geográfica es muy fácil publicitar la ruta en ferias internacionales e incrementar el número de visitantes extranjeros. Entre los factores negativos se encuentra la existencia de una oferta desigual, habiendo zonas muy concentradas de actividad (bodegas, restaurantes, etc.), mientras que en otros municipios de la ruta prácticamente no existe nada; asimismo, habría que controlar los estándares de calidad en toda la ruta, ya que algunas empresas asociadas son excelentes, pero otras son deficientes.

7. Así pues, la demanda de turismo del vino es creciente y ha generado empleo en época de crisis, lo cual implica que las empresas tienen un futuro prometedor si invierten más en esta área con la finalidad de satisfacer este segmento turístico. Cabe apuntar que en la consolidación de esta clase de destinos turísticos es necesaria una correcta promoción tanto por parte de las diferentes administraciones públicas como de las propias empresas privadas.

8. De tal modo, se aboga por crear una red de rutas del vino en el nivel nacional e internacional, empezando por los dos países de la Península Ibérica, España y Portugal, lo que permitiría al turista apreciar los distintos caldos así como conocer sus procesos productivos, a la vez que se realiza turismo cultural entre dos naciones unidas en la misma península, forjando un vínculo de desarrollo rural entre zonas vitivinícolas limítrofes. 


\section{FuENTES CONSULTADAS}

Acevin (s/f). Rutas del Vino de España [en línea]. Asociación Española de Ciudades del Vino. Disponible en: www.wineroutesofspain.com

Armesto, X. A. y B. Gómez (2004). "Productos agroalimentarios de calidad, turismo y desarrollo local: el caso del Priorat”. Cuadernos Geográficos, 34, 83-94.

Barrera, E. (2006). "Rutas alimentarias. Estrategias culturales de desarrollo territorial”. Patrimonio Cultural y Turismo, 15, 67-86.

Barrera, E. y O. Bringas (2008). "Food Trails: Tourist Architectures Built on Food Identity”. Scienze Gastronomiche, 3, 56-61.

Beverland, M. (1998). "Wine Tourism in New Zealand - Maybe the Industry has got it Right”. International Journal of Wine Marketing, 10 (2), 24-33.

Brunori, G. y A. Rossi (2000). "Synergy and Coherence through Collective Action: Some insights from Wine Routes in Tuscany”. Sociologia Rurales, 40 (4), 409-423.

Bruwer, J. (2003). "South Africa Wine Routes: Some Perspectives on the Wine Tourism Industry's Structural Dimensions and Wine Tourism Product”. Tourism Management, 24, 423-435.

Carlsen, J. (2004). "A Review of Global Wine Tourism Research". Journal of Wine Research, 15 (1), 5-13.

Charters, S. y J. Ali-Knight (2002). "Who is the Wine Tourist?" Tourism Management, 23, 311-319.

Correia, L., M. J. Passos y S. Charters (2004). "Wine Routes in Portugal: A Case Study of the Bairrada Wine Route”. Journal of Wine Research, 15 (1), 15-25.

Egan, D. y A. Bell (2002). "Chilean Wines: a Successful Image”. International Journal of Wine Marketing, 14 (2), 33-42.

Fernández, M. y A. Puig (2002). "El papel del cooperativismo en el turismo rural de la Comunidad Valenciana”. CIRIEC-España, 41, 183-212.

Frochot, I. (2000). "Wine Tourism in France: A Paradox", en C. M. Hall et al. (eds.). Wine Tourism around the World: Development, Management and Markets. Oxford: Elsevier, 67-80.

Getz, D. (2000). Explore Wine Tourism, Management, Development and Destinations. Nueva York: Cognizant Communication Corporation.

Getz, D. y G. Brown (2006). “Benchmarking Wine Tourism Development”. International Journal of Wine Marketing, 18 (2), 78-97. 
El desarrollo turístico de zonas rurales en España a partir de la creación de rutas del vino: un análisis DAF0

Gilbert, D. C. (1992). “Touristic Development of a Viticultural Regions of Spain”. International Journal of Wine Marketing, 4 (2), 25-32.

Hall, C. M. y R. Mitchell (2000). "Wine Tourism in the Mediterranean: A Tool for Reestructuring and Development”. Thunderbird International Business Review, 42 (4), 445-465.

Hall, C. M., et al. (1998). "Wine Tourism and Network Development in Australia and New Zealand: Review, Establishment and Prospects”. International Journal and Wine Marketing, 9 (2/3), 5-31.

Hall, C. M., et al. (eds.) (2000). Wine Tourism around the World: Development, Management and Markets. Oxford: Elsevier.

Hashimoto, A. y D. Telfer (2003). "Positioning an Emerging Wine Route in the Niagara Region: Understanding the Wine Tourism Market and its Implications for Marketing”. Journal of Travel and Tourism Marketing, 14 (3/4), 61-76.

Jolley, A. (2002). The Wine Industry, Wine Tourism and Tourism in General. Melbourne: Centre for Strategic Economic Studies of Victoria University.

López, J. A. (2010). "Posibilidades de desarrollo del enoturismo en la denominación de origen Jerez-Xerry y manzanilla de Sanlúcar de Barrameda y vinagre de Jerez". Boletín de la Asociación de Geógrafos Españoles, 53, 21-41.

Marques, H. (2006). "Research Report: Searching for Complementaries between Agriculture and Tourism: The Demarcated Wine-Producing Regions of Northern Portugal”. Tourism Economics, 12 (1), 147-161.

Martínez-Carrasco, L., M. Brugarolas y F. J. del Campo (2005). "Vinos de calidad poco conocidos: estimación de su potencial de mercado”. Revista Europea de Dirección y Economía de la Empresa, 14 (4), 139-156.

Mascarenhas, R. y J. M. Gândara (2010). "Producción y transformación territorial. La gastronomía como atractivo turístico”. Estudios y Perspectivas en Turismo, 19 (5), 776-791.

Melián, A., G. Millán y T. López-Guzmán (2008). "Vinos con denominación de origen: la Denominación de Origen Alicante”. Viticultura y Enología Profesional, 114, 5-15.

Millán, G. y A. Melián (2008). "Rutas turísticas enológicas y desarrollo rural. El caso estudio de la denominación de origen Montilla-Moriles en la provincia de Córdoba”. Papeles de Geografía, 47/48, 159-170. 
Mitchell, R. y C. M. Hall (2006). “Wine Tourism Research: The State of Play”. Tourism Review International, 9 (4), 307-332.

O’Neill, M. y S. Charters (2000). "Service Quality at the Cellar Door: Implications from Western Australia's Developing Wine Tourism Industry”. Managing Service Quality, 10, 112-122.

Oliveira, U. y M. M. Rocha (2011). "La herencia de la gastronomía portuguesa en Brasil como un producto del turismo cultural”. Estudios y Perspectivas en Turismo, 20 (2), 404-424.

Ravenscroft, N. y J. van Westering (2001). "Wine Tourism, Culture and the Everyday: A Theoretical Note”. Tourism and Hospitality Research, 3 (2), 149-162.

Schlüter, R. y D. Thiel (2008). "Gastronomía y turismo en Argentina Polo gastronómico Tomás Jofré”. Pasos. Revista de Turismo y Patrimonio Cultural, 6 (2), 249-268.

Secretaría de Estado de Turismo (2000). Propuesta para la asistencia técnica para el desarrollo del estudio de definición del producto "Rutas del Vino de España". Madrid: Servicio de Publicaciones del Ministerio de Industria, Turismo y Comercio.

Sharples, L. (2002). "Wine Tourism in Chile. A Brave New Step for a Brave New World". International Journal of Wine Marketing, 14 (2), 43-53.

Szivas, E. (1999). “The Development of Wine Tourism in Hungary”. International Journal of Wine Marketing, 11 (2), 7-17.

Telfer, D. J. (2001). "Strategic Alliances along the Niagara Wine Route”. Tourism Management, 22, 21-30.

Tzmitra-Kalogianni, I., A. Papadaki y A. Alexaki (1999). "Wine Routes in Northern Greece: Consumer Perceptions”. British Food Journal, 101 (11), 884-892.

Williams, P. W. y J. Kelly (2001). "Cultural Wine Tourists: Product Development Considerations for British Columbia's Resident Wine Tourism Market”. International Journal of Wine Marketing, 13 (3), 59-76. 